\title{
Stability-Indicating method for determination of Tizanidine Hydrochloride by LC-CAD method using chemometric approach
}

\author{
Mariana Brandalise $e^{\mathrm{a}}$; Pamela C. L. Ferreira ${ }^{\mathrm{a}}$; Leonardo Meneghinia ${ }^{\mathrm{a}}$ Andrea G. Pereira ${ }^{\mathrm{a}^{*}}$; \\ Pedro E. Fröelicha; Ana M. Bergold \\ ${ }^{a}$ Programa de Pós-Graduação em Ciências Farmacêuticas, Faculdade de Farmácia, Universidade Federal do Rio \\ Grande do Sul, Porto Alegre, Brasil \\ * Corresponding author e-mail: andreagp.far@gmail.com
}

\begin{abstract}
A new LC method for tizanidine hydrochloride in tablet dosage form using a charged aerosol detector (CAD) is described. The influence of various parameters on chromatographic system was investigated by factorial designs and Derringer's desirability. Chromatographic conditions were: mobile phase constituted of acetonitrile and ammonium acetate buffer $17 \mathrm{mM}(60: 40 ; \mathrm{v} / \mathrm{v})$, column oven at $39{ }^{\circ} \mathrm{C}$ and flow rate $0.8 \mathrm{~mL} \cdot \mathrm{min}^{-1}$ performed on Acclaim Trinity P1 column UV at $230 \mathrm{~nm}$. Thus, it was possible to validate a simple method to assay the tizanidine and its counter-ion in three formulations (drug reference, generic and manipulated). The present method showed specificity when challenged by forced degradation and excipients. Finally, the method was compared with USP monograph method demonstrating equivalence in assay evaluation.
\end{abstract}

Keywords: tizanidine hydrochloride, CAD detector, chemometrics, stability

\section{Introduction}

Tizanidine hydrochloride (TZ) (Figure 1), a central alpha-2 adrenoceptor agonist, is a myotonolytic agent used in the treatment of spasticity in patients with cerebral or spinal injury (dosages of 2 to $36 \mathrm{mg} / \mathrm{day}$ ) $(1,2)$.

United States Pharmacopeia (USP) have analytical methodologies involving TZ, using high performance liquid chromatography (LC) for related compounds determination (USP 39, 2016). Studies using LC have been reported with tizanidine alone or in association with other drugs, but there isn't one using association with charged aerosol detector (CAD) (2,5-8).

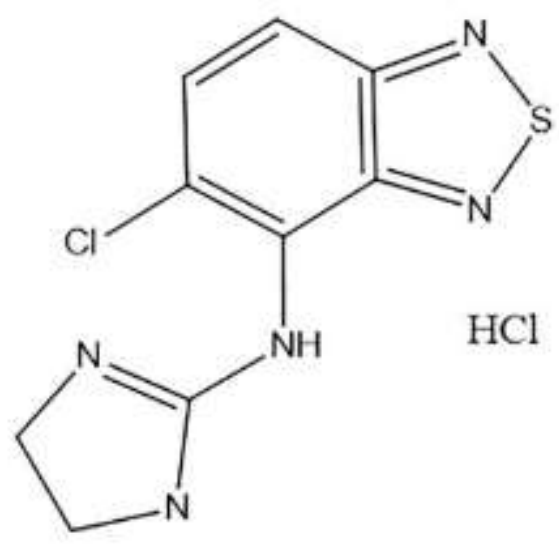

Figure 1 Structure of tizanidine hydrochloride.
CAD detector is a new type of detector introduced for LC applications. It recently began to be widely used in pharmaceutical analysis $(9,10)$. It has been compared to other detectors, such as the ELSD (evaporative light scattering detector), RI (refractive index), UV (ultraviolet) and MS (mass spectrometry), and has been found to be more sensitive and reproducible than ELSD and to exhibit more uniform response factors $(7,11-13)$. CAD is able to analyze a wider range of analytes than the UV detector due to its ability to detect non-chromophoric compounds, including counter-ions of drugs (14). As well as methods used to UV is necessary to optimize the chromatographic system to obtain the best detector response(9,15). A strategy involving Design of Experiments (DOE) is appropriate for use in the method development and validation stage for multiple factors and responses (16-18). Firstly, fractional factorial designs can be performed with many factors and to define those that are significant in the system. Further, a central composite design (CCD) is often used to obtain the optimum conditions for the responses. Additionally fractional factorial designs are used to assess the robustness parameter $(19,20)$.

This paper aimed to develop and validate a simple stability-indicating LC-CAD method to assay tizanidine hydrochloride in tablets. The counter-ion (chloride) was used for the identification and indirect quantification of the drug. Optimum chromatographic conditions were estimated by a face centered CCD and Derringer's desirability function. Three commercially available formulations were used: tablets reference (REF), tablets generic (GEN), and manipulated capsules (MAN). 


\section{Experimental}

Tizanidine hydrochloride $(99.70 \%)$ was acquired from Elder Pharmaceuticals Ltd. (India). Acetonitrile LC grade was from J. T Baker (USA); ammonium chloride and acetic acid were obtained from Synth (Brazil). All formulations, reference and generic drug tablets and manipulated capsules, containing $2 \mathrm{mg}$ of tizanidine hydrochloride were purchased in the Brazilian market. The filter units were purchased from Millipore ${ }^{\circledR}$ (USA). Water was purified with the Milli$\mathrm{Q}^{\circledR}$ Plus system (Milipore Corp., Billerica, MA).

LC-CAD method was developed and validated using Shimadzu chromatoghapic system using LC20AT binary pump. In all experiments, Corona CAD (Thermo Scientific, Waltham, MA USA) was connected in series with SPD-AVvp UV/VIS photodiode array detector, SIL 20-AC autosampler and CTO20A oven. UV/VIS and CAD signals were obtained simultaneously. Chromatographic runs were performed an Acclaim Trinity P1 (C18 column; $3.0 \mathrm{~mm}$ x $100 \mathrm{~mm}, 3 \mu \mathrm{m}$ ), $\mathrm{CAD}$ has a nitrogen generator and was used as the nebulizer gas for the CoronaCAD at a pressure of 35 psi, range $100 \mathrm{pA}$. The wavelength established for UV detector was $230 \mathrm{~nm}$. Injection volume for all the analyses was set at $20 \mu \mathrm{L}$.

\section{Softwares}

Design-Expert ${ }^{\circledR} 7.0 .0$ version (Stat-Ease Inc., USA) was used for DOE analysis. Statistical analysis for the validation was carried out by MINITAB ${ }^{\circledR}$, version 15.0 (Stat-Soft Inc., USA).

\section{Method Development}

Initially, reversed-phase columns were tested and it wasn't able to separate TZ and its degraded products. In this way, a column with mixed groups (octadecylsilane and cationic/ anionic clusters) in stationary phase was tested and the analytes showed adequated peack resolution and with retention time lower than obtained in others methods with run time of $20 \mathrm{~min}$. Therefore, a fractional factorial design $2^{5-2}$ was performed to access significant factors that affected the retention factor $(k)$ of tizanidine and its counter-ion chloride. Fractional designs are expressed using the notation $K^{l-n}$, where $k$ is the number of levels (low level, -1; high level, +1) of each factor investigated, $l$ is the number of factors investigated, and $n$ the size of the fraction of the full factorial used. A $2^{5-2}$ design is $1 / 4$ of a twolevel, five-factor factorial design, but with only eight runs (experiments). Later, the significant factors studied in $2^{5-2}$ design were applied in a face-centered CCD. In this design, besides k', four more responses were analyzed: resolution (impurity and degradation product obtained from stress studies) and limit of quantification (for tizanidine and chloride). These responses were modeled by polynomial models.

For an experimental design with three factors, a loworder polynomial model is usually employed:

$y=b_{0}+b_{1} x_{1}+b_{2} x_{2}+b_{3} x_{3}+b_{12} x_{1} x_{2}+b_{13} x_{1} x_{3}+b_{23} x_{2} x_{3}$

In this first order response surface model, $y$ represents the estimated response (e.g., resolution); $b_{0}$, $b_{1}, b_{2}, b_{\mathrm{m}}$ is a set of unknown parameters where $b_{0}$ is the average experimental response, $b_{1}$ to $b_{3}$ are the estimated effects (main effects) on the factors $\left(\mathrm{x}_{1}, \mathrm{x}_{2}, \mathrm{x}_{3}\right)$ and $b_{12}$ to $b_{23}$ are estimated effects with interaction. After model assessment a higher order (quadratic or cubic) polynomial model and/or mathematical transformation in the response is sometimes necessary (21).

Subsequently functions obtained from individual responses were used in Derringer's desirability. First step in this procedure was to convert each response into an individual desirability function $(d i)$ which varies over the range of $0 \leq \mathrm{di} \geq 1$, where $d i=1$ is the best alternative and $d i=0$ when the response is outside the optimal region. The design variables were then chosen to maximize global desirability $(D)$,

$$
D=\left(\mathrm{d}_{1} \times \mathrm{d}_{2} \times \ldots \mathrm{d}_{\mathrm{m}}\right)^{1 / \mathrm{m}}
$$

where $m$ standards responses and $d 1, d 2 \ldots d_{m}$ are the individual desirabilities. If the objective or target $T$ for the response $y$ is a maximum value, when weight $r=1$, the desirability function is linear. Choosing $r>1$ places more emphasis on being close to the target value, and choosing $0<r<1$ makes this less important. If the target for the response is a minimum value,

$$
\left\{\begin{array}{c}
0, \quad y<L \\
\mathrm{~d}=\left(\frac{y-L}{T-L}\right)^{r} L \leq \mathrm{y} \leq \mathrm{T} \\
1, \quad \mathrm{y}>\mathrm{T}
\end{array}\right.
$$

the two-sided desirability function (4.0) assumes that the target is located between the lower (L) and the upper (U) limits, and is defined as

$$
\left\{\begin{array}{cc}
1, & y<T \\
d= & \left(\begin{array}{c}
U-y \\
U-T
\end{array}\right)^{r} T \leq y \leq U \\
0, & \begin{array}{r}
y>U \\
0.0)
\end{array}
\end{array}\right.
$$

After building the function, input and output values were selected. Finally, experimental values were compared with theoretical (predicted) values to check model adequacy and predictive power. 


$$
\mathrm{d}=\left\{\begin{array}{l}
0, \quad \mathrm{y}<\mathrm{L} \\
\left(\frac{y-L}{T-L}\right)^{r 1} \quad \mathrm{~L} \leq \mathrm{y} \leq \mathrm{T} \\
\left(\frac{U-y}{U-T}\right)^{r 2} \quad \mathrm{~L} \leq \mathrm{y} \leq \mathrm{U} \\
0, \\
\mathrm{y}>\mathrm{U}
\end{array}\right.
$$

\section{Validation}

\section{System suitability and solution stability}

Stability of TZ in solution was assessed at room temperature and in refrigerated conditions. Prior to beginning the method validation stage the accuracy and precision of LC data collected were checked.Ten injections were performed and the chromatographic parameters were evaluated with RSD.

\section{Specificity and Stress study}

Excipients from three formulations were obtained by donation from local pharmacy. Pools from these were diluted in mobile phase (acetonitrile: $17 \mathrm{~m} M$ ammonium acetate $(60: 40 ; \mathrm{v} / \mathrm{v}))$ and analyzed by the proposed method to verify the presence of interferences.

Additionally, forced decomposition was performed according to the guidelines and specific literature (22) in water solutions containing drug substance or drug product. Intentional degradation was carried out by exposing $5 \mathrm{~mL}$ of reference/test stock solution to $5 \mathrm{~mL}$ of acetic acid $0.5 \mathrm{M}$ or ammonium hydroxide $0.5 \mathrm{M}$. Solutions were withdrawn from a $10 \mathrm{~mL}$ volumetric flask, allowed to reach room temperature and then neutralized with acid or base (when necessary). Oxidative degradation was performed by mixing equal volumes of standard stock solution with $13 \%$ hydrogen peroxide solution in a $10 \mathrm{~mL}$ volumetric flask. Samples were allowed to reach ambient temperature and diluted with water. Solutions were kept in a dry oven at $70^{\circ} \mathrm{C}$ for different lengths of time to perform a thermal stress study. Photolytic studies were carried out with a $2 \mathrm{~mL}$ sample in a quartz container and exposed to light (UV $352 \mathrm{~nm}$ ) in a photostability chamber (controlled temperature). Blank solutions were prepared by the aforementioned procedure, using water instead of stock solutions. Analytical data of the method were collected by LC-PDA-CAD detector.

The method was optimized using the degraded samples.

\section{Linearity and range}

Solutions of $\mathrm{TZ}$ were prepared at six concentrations (4.0, 8.0, 12.0, 24.0, 32.0, and $40.0 \mu \mathrm{g} / \mathrm{mL})$ spanning a range of $20-200 \%$ of the target $\mathrm{TZ}$ assay concentration (20 $\mu \mathrm{g} / \mathrm{mL})$, and chloride solutions were prepared at five concentration $(3.0,5.0,7.0,9.0$ and $11 \mu \mathrm{g} / \mathrm{mL})$ for linearity experiments. Ordinary least-squares method was applied to calibration curve construction $y=a x \pm b$, where $y$ is the response (peak area), $x$ is the concentration, and $a$ and $b$ are the slope and intercept, respectively. Model was evaluated by determination coefficient regression significance, lack-of-fit and residual analysis.

\section{Precision}

Twenty tablets from each formulation(REF, GEN and MAN) were accurately weighed and finely powdered. A portion of powder equivalent to $0.4 \mathrm{mg}$ of tizanidine was accurately weighed and transferred to a $20 \mathrm{~mL}$ volumetric flask, placed for 20 minutes in an ultrasonic bath and diluted with water. Then, the sample solutions were filtered through $0.45 \mu \mathrm{m}$ PVDF membrane. Seven experiments (of each sample) were carried out in triplicate on three different days. The RSD was assessed for repeatability and intermediate precision.

\section{Accuracy}

Method accuracy was calculated as a percentage of recovery by assaying the known added amount of analyte in the samples. The concentrations were $75 \%$, $100 \%$ and $125 \%$ of nominal concentration.

\section{Limit of detection (LOD) and limit of quantification (LOQ)}

LOQ was determined based on signal-to-noise ratio $\left(\mathrm{R}_{\mathrm{S} / \mathrm{N}}\right)$, with 10:1 ratio. This limit was subsequently validated by the assay of a set of samples. Likewise, LOD was assessed on $\mathrm{R}_{\mathrm{S} / \mathrm{N}}$ with a $3: 1$ ratio.

\section{Robustness}

A Plackett-Burman design was constructed with six $(A-F)$ factors performed in 13 runs. Factors named dummy represent unreal physical changes. Nominal levels (" 0 " level) are the conditions used in the method validation. Three responses were evaluated: peak asymmetry of $\mathrm{TZ}$ and chloride $(\mathrm{Cl})$, and $\mathrm{TZ}$ content by tablet assay. Only the reference formulation was used. The Pareto chart (with $t$ statistics) was used to access relevant effects.

\section{Method Comparison}

The validated LC-CAD method was compared with method from USP monograph of TZ(3). This method uses $\mathrm{C}_{18}$ column, wavelength $237 \mathrm{~nm}, 1.0 \mathrm{~mL} \cdot \mathrm{min}^{-1}$ flow rate, mobile phase with acetonitrile and buffer solution $(\mathrm{pH} 3.0)$ at a 80:20 ratio and a column maintained at temperature $\left(50{ }^{\circ} \mathrm{C}\right)$. The comparison was performed between the reference formulation and $t$-test was used to access significant difference between the methods.

\section{Results and Discussion}

Method Development and Forced Degradation 
Initial screening using a $2^{5-2}$ design showed that the lowest $k^{\prime}$ values (tizanidine and its counter-ion) were found when significant factors (organic solvent type and content, buffer concentration, flow rate, oven temperature) were at "high" levels. No considerations concerning interactions are possible because of the low design resolution. Mobile phase that showed appropriate results were acetonitrile with ammonium acetate buffer. A face-centered $C C D$ was applied using acetonitrile concentration, ammonium acetate concentration and column oven temperature.

Initially samples from stress study were analyzed using center point design to access which responses should be optimized. Samples in $\mathrm{H}_{2} \mathrm{O}_{2} 13 \%$ for $72 \mathrm{~h}$ (Table 1) presented significant degradation which was analyzed with full CCD. For retention factor, $\mathrm{TZ}\left(\mathrm{K}_{\mathrm{TZ}}\right)$ showed the influence of 3 factors, but chloride $\left(\mathrm{K}_{\mathrm{Cl}}\right)$ was only influenced by buffer concentration and acetonitrile content. It is suggested that tizanidine hydrochloride, in dissociative state, presented interaction with both hydrophobic and hydrophilic sites in a column, as demonstrated for others drugs (14). Thus, when organic content and ionic strength are increased, column retention is reduced and consequently retention time too. This is probably unrelated to the acetonitrile elution strength, but rather due to the influence of organic solvent on the dissociation of ions from amonium acetate and solvation power of mobile phase on the chloride (23). In this way, the acetonitrile content provides an inverse effect on $\mathrm{K}_{\mathrm{cl}}^{\prime}$ when compared with $\mathrm{K}_{\mathrm{TZ}}$ (Figure 2, B). In the case of resolution between degradation product and tizanidine $\left(R_{D}\right)$, all runs provided good separation since degradation product do not co-elute with TZ.

Table 1 Results of forced degradation study using optimized method for tizanidine tablets.

\begin{tabular}{|c|c|c|c|c|c|}
\hline \multirow{2}{*}{$\begin{array}{l}\text { Stress } \\
\text { Agent }\end{array}$} & \multirow{2}{*}{$\begin{array}{c}\text { Time } \\
\text { (hours) }\end{array}$} & \multicolumn{4}{|c|}{ Assay (\%) } \\
\hline & & Standart $^{\mathrm{a}}$ & $\begin{array}{c}\text { Reference } \\
\text { Drug }\end{array}$ & $\begin{array}{c}\text { Generic } \\
\text { Drug }\end{array}$ & $\begin{array}{c}\text { Manipulated } \\
\text { Drug }\end{array}$ \\
\hline $\mathrm{H}_{2} \mathrm{O}_{2} 13 \%$ & 24 & 100.36 & 100.42 & 99.69 & 99.20 \\
\hline $\mathrm{H}_{2} \mathrm{O}_{2} 13 \%$ & 48 & 100.09 & 99.87 & 100.02 & 99.09 \\
\hline $\mathrm{H}_{2} \mathrm{O}_{2} 13 \%$ & 72 & 92.58 & 93.36 & 94.11 & 94.50 \\
\hline $\begin{array}{l}\text { Acetic Acid } \\
0.5 M\end{array}$ & 72 & 99.83 & 100.01 & 100.74 & 99.71 \\
\hline $\mathrm{NH}_{4} \mathrm{OH}$ & 72 & 100.25 & 100.90 & 99.74 & 100.37 \\
\hline Heat & 72 & 99.75 & 100.05 & 99.26 & 99.60 \\
\hline UV $352 \mathrm{~nm}$ & 72 & 100.37 & 99.49 & 99.90 & 100.80 \\
\hline
\end{tabular}

Ionic strength was critical because at some levels it helped the separation (narrower peak, information not shown) and at others reduced the retention until inadequate separation with tizanidine and others peaks. Additionally, the limit of quantification for tizanidinine $\left(L Q_{T Z}\right)$ was affected by acetonitrile concentration and buffer concentration.

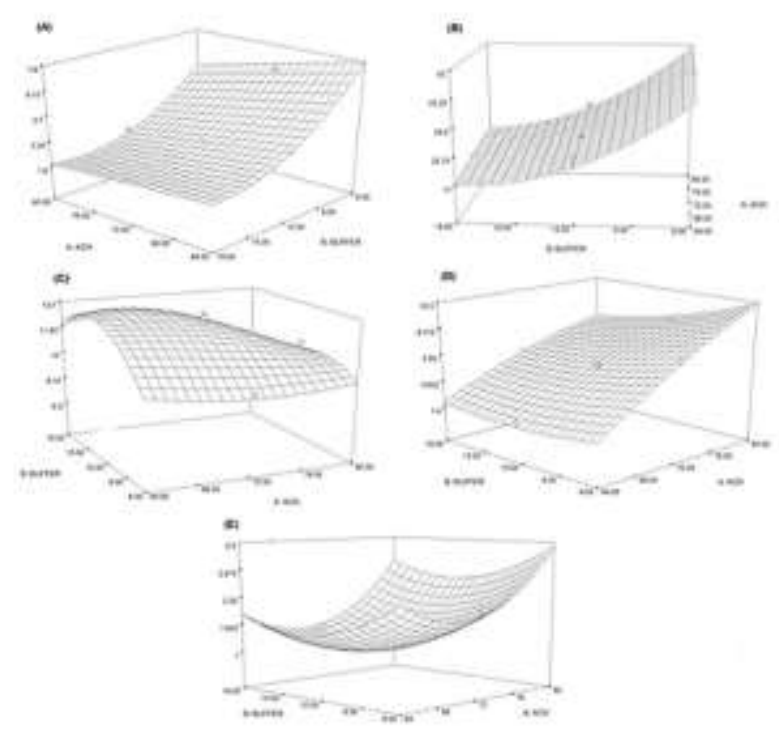

Figure 2 Response surfaces for modeled responses, where $A$ is factor retentions of tizanidine, $B$ is factor retention of chloride, $C$ is resolution peak degradation, $D$ is resolution peak sodium and $E$ limit of quantification for tizanidine.

For $L Q_{C}$ only amonium acetate affected this parameter probably because peak shape is more related to hydrophilic interactions with cationic/aninonic column groups than hydrophobic docadecylsilane.

In RSMs (Figure 2), it can be observed that the responses present different profiles, and it is necessary to perform Derringer's desirability for global optimization. Several attempts were performed, until a possible and optimized system was obtained. The final values used for method validation were a mobile phase with acetonitrile and $17 \mathrm{mM}$ ammonium acetate (60:40; $\mathrm{v} / \mathrm{v})$, column oven at $39{ }^{\circ} \mathrm{C}$ and flow rate $0.8 \mathrm{~mL} \cdot \mathrm{min}^{-1}$.

\section{Validation}

Based on the optimization results, method was validated and the results are shown in table 1. Samples were stable for 30 days under refrigeration and 3 days at room temperature (in mobile phase solution, information not shown).

Specificity challenged with excipients (pool) and samples submitted to forced degradation presented no interference at the tizanidine or chloride peak (Figure 3). Degradation rate was the same observed when the degraded samples were analyzed by center point from CCD (Table 1). Also, the peak that probably originated from degradation is not observed in a photodiode array, an advantage when CAD detection is used. Moreover, robustness does not show significant differences when the method is carried out with the proposed changes. 


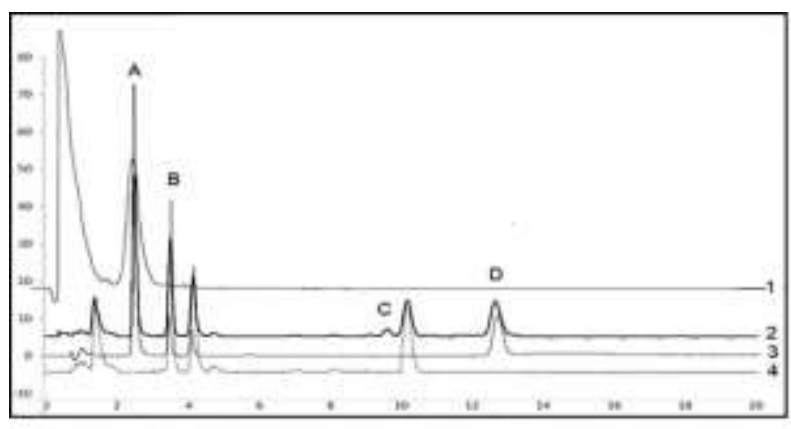

Figure 3 Chromatogram showing REF tablets from oxidative stress, detector UV (230 nm) (1), REF tablets from oxidative stress, detector CAD (2), SQR, detector CAD (3) and blank (hydrogen peroxide $13 \%+$ excipients), detector $\mathrm{CAD}(4)$ and (A) peak tizanidine; (B) peak Interfering; (C) degradation product and (D) Chloride.

\section{Method Comparison}

Results from comparison between LC-CAD method and USP method are demonstrated in Table 2. No significant difference was found and LC-CAD can be considered equivalent to the USP method.

Table 2 Comparison of the method validated with USP 34 method for tizanidine tablets.

\begin{tabular}{|c|c|c|c|c|c|c|}
\hline \multirow{2}{*}{$\begin{array}{l}\text { Amount } \\
\text { Method }\end{array}$} & \multicolumn{2}{|c|}{ Reference } & \multicolumn{2}{|c|}{ Generic } & \multicolumn{2}{|c|}{ Manipulated } \\
\hline & CAD & USP & CAD & USP & CAD & USP \\
\hline Mean\% & 97.47 & 97.49 & 97.23 & 97.68 & 100.34 & 99.96 \\
\hline \multicolumn{7}{|l|}{ assay } \\
\hline RSD & 1.19 & 1.31 & 1.37 & 1.54 & 1.29 & 1.40 \\
\hline \multicolumn{7}{|l|}{$(\%)$} \\
\hline \multicolumn{7}{|c|}{ Paired t-test } \\
\hline Amount & \multicolumn{2}{|c|}{ Reference } & \multicolumn{2}{|c|}{ Generic } & \multicolumn{2}{|c|}{ Manipulated } \\
\hline p-value* & \multicolumn{2}{|r|}{0.864} & \multicolumn{2}{|c|}{0.069} & \multicolumn{2}{|c|}{0.128} \\
\hline
\end{tabular}

\section{Conclusions}

Stability-indicating LC-CAD method was successfully developed and validated. The chemometric methodologies employed were useful to improve information about the influence of the factors on responses. Comparison between different detectors showed different chromatographic profiles and it is suggested an advantage to CAD detection because it was able to detect and quantify the drug and its counterion (chloride) in the same analytical run. A robustness study provided a more complete evaluation of deliberate variations in the method. The developed method demonstrated to be equivalent to the USP assay method. Therefore, it is suggested to the routine assay analysis of drug products.

\section{Acknowledgements}

The authors thank CAPES and CNPq of Brazil.

\section{References}

1. Qi, M.L., Wang, P., Wang, L. Validated liquid chromatography method for assay of tizanidine in drug substance and formulated products. Anal Chim Acta. 2003;478(2):171-7.

2. Nimje, H., Wate, S.P., Dharkar, D.P., Razdan, R. Simultaneous RPHPLC determination of nimesulide and tizanidine in tablets. Indian J Pharm Sci [Internet]. 2007;69(2):281-3. Available from: http://www.ijpsonline.com/text.asp?2007/69/2/281/3315 8

3. USP 39. The United States Pharmacopeial Convention. NF 39 Rockv United States Pharmacopeial. 2016;

4. The Pharmacopoeia of Japan 15.ed. Tokyo: Society of Japanese Pharmacopoeia. 2006;1179.

5. Puranik, M., Wadher, S., Dhole, S., Yeole, P. Simultaneous Estimation of Valdecoxib and Tizanidine Hydrocloride in tabelts by RP-HPLC. Indian J Pharm Sci. 2006;68:670-2.

6. Vaidya, V.V., Singh, G.R., Choukekar, M.P., Kekare, M.B. Simultaneous RP HPLC Determination of Aceclofenac, Paracetamol and Tizanidine in Pharmaceutical Preparations. E-Journal Chem [Internet]. 2010;7(1):260-4. Available from: http://www.hindawi.com/journals/chem/2010/323410/

7. Ramaa, C., Deshpande, D., Shirode, A., Wamorkar, V., Kakad, A., Kadam, V. Reverse-phase high performance liquid chromatographic determination of Tizanidine and Valdecoxib in tablets. Indian $\mathrm{J}$ Pharm Sci [Internet]. 2006;68(4):514. Available from: http://www.ijpsonline.com/text.asp?2006/68/4/514/2783 2

8. Mahadik, K.R., Paradkar, A.R., Agrawal, H., Kaul, N. Stability-indicating HPTLC determination of tizanidine hydrochloride in bulk drug and pharmaceutical formulations. J Pharm Biomed Anal. 2003;33(4):545-52.

9. Dixon, R.W., Peterson, D.S. Development and Testing of a Detection Method for Liquid Chromatography Based on Aerosol Charging. 2002;74(13):2930-7.

10. Vehovec, T., Obreza, A. Review of operating principle and applications of the charged aerosol detector. J Chromatogr A [Internet]. 2010 Mar 5 [cited 2014 Nov 12];1217(10):1549-56. Available from: http://www.ncbi.nlm.nih.gov/pubmed/20083252

11. Takahashi, K., Kinugasa, S., Senda, M., Kimizuka, K., Fukushima, K., Matsumoto, T. et al. Quantitative comparison of a corona-charged aerosol detector and an evaporative light-scattering detector for the analysis of a 
synthetic polymer by supercritical fluid chromatography. J Chromatogr A. 2008;1193(12):151-5.

12. Vervoort, N., Daemen, D., Török, G. Performance evaluation of evaporative light scattering detection and charged aerosol detection in reversed phase liquid chromatography. J Chromatogr A [Internet]. 2008 May;1189(1-2):92-100.

Available

from:http://linkinghub.elsevier.com/retrieve/pii/S00219 67307018985

13. Kou, D., Manius, G., Zhan, S., Chokshi, H.P. Size exclusion chromatography with Corona charged aerosol detector for the analysis of polyethylene glycol polymer. J Chromatogr A [Internet]. 2009 Jul;1216(28):5424-8. Available

from: http://linkinghub.elsevier.com/retrieve/pii/S0021967309 007705

14. Zhang, K., Dai, L., Chetwyn, N.P. Simultaneous determination of positive and negative pharmaceutical counterions using mixed-mode chromatography coupled with charged aerosol detector. J Chromatogr A [Internet]. 2010 Sep;1217(37):5776-84. Available from: http://linkinghub.elsevier.com/retrieve/pii/S0021967310 009556

15. Górecki, T., Lynen, F., Szucs, R., Sandra, P. Universal Response in Liquid Chromatography Using Charged Aerosol Detection. Anal Chem [Internet]. 2006 May;78(9):3186-92. Available from: http://pubs.acs.org/doi/abs/10.1021/ac060078j

16. Leardi, R. Experimental design in chemistry: A tutorial. Anal Chim Acta. 2009;652(1-2):161-72.

17. Kazakevich, Y., Lobrutto, R. HPLC for Pharmaceutical Scientists. Kazakevich Y, Lobrutto R, editors. New Jersey: Wiley; 2007.

18. Montgomery, D.C. Design and Analysis of Experiments Eighth Edition. 8th ed. John Wiley \& Sons, editor. New Jersey: Wiley; 2000.

19. ICH. International Conference on HarmonisationHarmonised Tripartite Guideline: Validation of Analytical Procedures: text and methodology, Q2(R1). In 2005.

20. Dejaegher, B., Heyden, Y.V. Ruggedness and robustness testing. J Chromatogr A [Internet]. 2007 Jul;1158(1-2):138-57. Available from: http://linkinghub.elsevier.com/retrieve/pii/S0021967307 003950

21. Mason, R.L., Gunst, R.F., Hess, J.L. Statistical Design and Analysis of Experiments [Internet]. 2nd ed. Hoboken, NJ, USA: John Wiley \& Sons, Inc.; 2003. Available http://doi.wiley.com/10.1002/0471458503
22. Myers, R.H., Montgomery, D.C., Anderson-Cook, C.M. Response Surface Methodology: Process and Product Optimization Using Designed Experiments. 3rd ed. New Jersey: Wiley; 2003.

23. Sýkora, D., Tesařová, E., Popl M. Interactions of basic compounds in reversed-phase high-performance liquid chromatography influence of sorbent character, mobile phase composition, and $\mathrm{pH}$ on retention of basic compounds. J Chromatogr A [Internet]. 1997 Jan;758(1):37-51. Available from: http://linkinghub.elsevier.com/retrieve/pii/S0021967396 006917 